\title{
artigo
}

\section{Elaboração de instrumento de coleta de dados para o exame físico do aparellho respiratório}

\author{
Elaboration of data collection instrument for physical examination of respiratory system \\ Elaboración del instrumento de recogida de datos para el examen físico del sistema respiratorio
}

\begin{abstract}
RESUMO
Objetivo: Descrever o desenvolvimento de um instrumento de coleta de dados para exame físico do aparelho respiratório destinado a enfermeiros e acadêmicos de enfermagem. Metodologia: Estudo metodológico desenvolvido em 2019 em duas etapas: seleção do conteúdo na literatura nacional e internacional e construção do instrumento com foco no processo de enfermagem e exame do aparelho respiratório. Resultados: A tecnologia denominada "Guia para o Exame Físico do Sistema Respiratório" ficou organizado em sete seções: preparo inicial; identificação do paciente; entrevista de enfermagem; história pregressa; história pessoal; exame físico; necessidades humanas básicas (NHB) sugeridas; título diagnóstico de enfermagem de Nanda internacional 2018-2020, sugeridos; anotações do examinador. Considerações Finais: A tecnologia educativa é relevante para a coleta de dados durante a execução da primeira etapa do processo de enfermagem.
\end{abstract}

DESCRITORES: Tecnologia em Saúde; Exame Físico; Sistema Respiratório.

\section{ABSTRACT}

Objective: To describe the development of a data collection instrument for physical examination of the respiratory tract for nurses and nursing students. Methodology: Methodological study developed in 2019 in two stages: selection of content in national and international literature and construction of the instrument focusing on the nursing process and examination of the respiratory tract. Results: The technology called "Guide for Physical Examination of the Respiratory System" was organized into seven sections: initial preparation; patient identification; nursing interview; past history; personal history; physical exam; suggested basic human needs (NHB); Nanda international nursing diagnostic title 2018-2020, suggested;eExaminer's notes. Final Considerations: Educational technology is relevant for data collection during the execution of the first stage of the nursing process.

DESCRIPTORS: Health Technology; Physical Exam; Respiratory System.

\section{RESUMEN}

Objetivo: Describir el desarrollo de un instrumento de recolección de datos para la exploración física del aparato respiratorio para enfermeras y estudiantes de enfermería. Metodología: Estudio metodológico desarrollado en 2019 en las etapas: selección de contenidos en la literatura nacional e internacional y construcción del instrumento con foco en el proceso de enfermería y exploración del sistema respiratorio. Resultados: La tecnología denominada "Guía para la exploración física del aparato respiratorio" se organizó en siete apartados: preparación inicial; identificación del paciente; entrevista de enfermería; historia pasada; Historia personal; examen físico; necesidades humanas básicas (NHB) sugeridas; Título de diagnóstico de enfermería de Nanda internacional 2018-2020, sugerido; notas del examinador. Consideraciones Finales: La tecnología educativa es relevante para la recolección de datos durante la ejecución de la primera etapa del proceso de enfermería.

DESCRIPTORES: Tecnología de la Salud; Examen Físico; Sistema Respiratorio.

RECEBIDO EM: 07/06/2020 APROVADO EM: 07/06/2020

\author{
Hevellyn Andrade Faller Régino \\ Enfermeira. Pós-Graduada em Enfermagem em Terapia Intensiva Neonatal e Pediátrica. ES, Brasil. \\ ORCID: 0000-0003-3260-8523
}

\section{Marcelo Costa Vicente}

Enfermeiro, mestre em saúde coletiva - Faculdades Integradas São Pedro.

ORCID: 0000-0002-5518-2900 


\section{Maristela Villarinho de Oliveira}

Enfermeira docente do Centro Universitário Salesiano.

ORCID: 0000-0002-5265-9195

\section{Rozeli Brandão da S. M. Leite}

Enfermeira do Hospital Universitário Cassiano Antônio de Moraes HUCAM.

ORCID: 0000-0002-3086-3136

\section{Livia Perasol Bedin}

Enfermeira. Doutora em enfermagem e docente do Centro Universitário Salesiano. ORCID: 0000-0001-8228-6468

\section{Claudia Curbani Vieira Manola}

Enfermeira mestre em administração e docente do Centro Universitário Salesiano. ORCID: 0000-0002-0925-4432

\section{Evandro Bernardino Mendes de Melo}

Enfermeiro. Doutorando em Enfermagem na Universidade Federal de Minas Gerais. MG, Brasil e Docente do Centro Universitário Salesiano.

ORCID: 0000-0002-1772-3083

\section{INTRODUÇÃO}

0 processo de respiração compreende uma das funções vitais para o ser humano, pois é, por meio dela, que o processo de hematose se consolida promovendo a eliminação do gás carbônico dentre outras substâncias, e a captação do oxigênio fundamental para a homeostase do organismo ${ }^{(1)}$.

Sabe-se que as alterações desse sistema pode ser evidenciadas por meio da coleta de dados composta pela entrevista e exame físico de enfermagem, na qual promove a oportunidade ideal para avaliação do paciente, uma vez que permite o registro de informações relatadas (dados subjetivos) e obtenção de achados clínicos por meio das técnicas propedêuticas de inspeção, palpação, percussão e ausculta (dados objetivo), trata-se do saber fazer da enfermagem, podendo ser entendida como tecnologia do cuidado ${ }^{(2-4)}$.

Quanto às tecnologias, é notório que tem trazido grandes contribuições para todas as áreas do conhecimento, entre elas a de saúde, e nela incluída, a enfermagem ${ }^{(5)}$.

Entende-se por tecnologia o conhecimento aplicado para a prevenção, diagnóstico e tratamento de doenças e reabilitação de suas consequências. Dentre os diversos saberes da enfermagem, o histórico e o exame físico de enfermagem fazem parte de uma tecnologia do cuidado, denomina- da Processo de Enfermagem, dividida em cinco etapas: coleta de dados que compreende o histórico e exame físico, diagnóstico, planejamento, implementação e avaliação de enfermagem ${ }^{(6,7)}$.

Sabe-se que é de suma importância que os estudantes de enfermagem e enfermeiros dominem a etapa de coleta de dados fundamentada em um referencial teórico, no Brasil, um dos referenciais mais conhecido, utilizado e aplicado, é a Teoria das Necessidades Humanas Básicas (NHB) de Wanda Horta, que permite uma visão holística do paciente e reforça a importância do cuidado ao ser humano em suas necessidades psicobiológicas, psicossociais e psicoespirituais. Sendo assim, entende-se a coleta de dados como a base do Processo de Enfermagem, que orienta o cuidado, auxilia no registro da assistência e reafirma o papel do enfermeiro quanto a sua cientificidade ${ }^{(8)}$.

Cabe ressaltar também que a coleta de dados está regulamentada pela Resolução n. ${ }^{\circ}$ 358/2009 do Conselho Federal de Enfermagem do Brasil, que a tornou obrigatória/compulsória em todo território nacional e em todos os cenários onde se dá o exercício clínico pelo enfermeiro. A referida resolução descreve que é de competência desse profissional o registro em prontuário das informações coletadas por meio do Processo de Enfermagem ${ }^{(9,10)}$.

Sabe-se que os estudantes de enferma- gem e enfermeiros possuem dificuldades em realizar a entrevista e o exame físico do aparelho respiratório, estudos apontam que a falta de habilidade teórico/prática é um dos principais fatores que dificulta a realização da entrevista e do exame físico e, consequentemente, impacta na operacionalização do PE, essencial para cientificidade e visibilidade da profissão. Em soma, outros fatores apontados pela literatura são: lacunas no processo de ensino aprendizagem; falta de habilidade técnica e científica; esquecimento do suporte teórico; dificuldade de aprender a codificação típica da área da saúde; medo; insegurança na verificação, interpretação dos achados e atribuição de nomenclatura específica ${ }^{(11,12)}$.

Nessa perspectiva, foi considerada a necessidade de criação de um instrumento voltado para coleta de dados que possa auxiliar o estudante e o enfermeiro na realização da entrevista e exame físico do aparelho respiratório e, consequentemente, o registro, compreendido como imprescindível no processo de cuidado humano, visto que, quando redigido de maneira que retrata a realidade a ser documentada, possibilita a comunicação permanente ${ }^{(13,14)}$.

Vale ressaltar que não basta somente coletar os dados por meio da entrevista de enfermagem e exame físico e registrá-los adequadamente, faz-se necessário a incorporação e uso das taxonomias de enfer- 


\section{artigo}

magem, para que a comunicação entre os enfermeiros ocorra de forma mais efetiva. Dentre elas, pode-se citar a Classificação da Associação Norte Americana de Diagnósticos de Enfermagem - NANDA Internacional (NANDA-I). A NANDA-I é a primeira organização a criar uma linguagem diagnóstica padronizada e própria da enfermagem na década de 1970 . A versão mais atual dessa classificação (2018-2020) está organizada em 13 domínios, 47 classes e 244 diagnósticos de enfermagem ${ }^{(15)}$.

Diante das questões apresentadas, a presente pesquisa teve por objetivo elaborar e apresentar um instrumento de coleta de dados do aparelho respiratório, a partir da Teoria das Necessidades Humanas Básicas e Títulos Diagnósticos da NANDA-I, para utilização de estudantes de enfermagem e enfermeiros.

\section{METODOLOGIA}

Estudo metodológico fundamentado em duas etapas: seleção do conteúdo na literatura nacional e internacional e construção do instrumento com foco no processo de enfermagem e exame do aparelho respiratório. Os estudos metodológicos visam à investigação de métodos para coleta e organização dos dados, tais como: desenvolvimento, validação e avaliação de ferramentas e métodos de pesquisa, o que favorece a condução de investigações com rigor acentuado ${ }^{(16)}$.

A seleção do conteúdo foi realizada por meio de consulta da produção nacional e internacional de enfermagem publicada sob forma de livros, artigos e diretrizes que contemplam a semiologia do aparelho respiratório. A primeira versão do instrumento derivou da coleta de dados das literaturas encontradas na biblioteca da Católica de Vitória Centro Universitário, entre julho e setembro de 2019. Os livros são demonstrados no Quadro 1:

Procurou-se, nessa revisão, identificar os conteúdos relacionados ao exame físico do aparelho respiratório associados às principais Necessidades Humanas Básicas (NHB) elencadas de acordo com os conteúdos do exame físico, em soma, também foram associados os principais títulos diagnósticos da Associação Norte Americana de Diagnóstico de Enfermagem NANDA-I (2018-2020).

Os conteúdos selecionados por meio da revisão de literatura serviram como base para elaboração do guia de exame físico do aparelho respiratório, no qual foi constituído sob a forma de checklist e espaços abertos para o examinador escrever suas observações, por sua vez, em sua estrutura, o guia foi elaborado por meio de sete sessões, sendo: 1) Preparo inicial; 2) Identificação do paciente; 3 ) Entrevista de Enfermagem; 4) Exame físico; 5) Necessidades Humanas Básicas sugeridas; 6) Títulos Diagnósticos de Enfermagem da NANDA-I; 7) Anotações do examinador.

Cabe ressaltar que pela especificidade do constructo, os conteúdos referentes à elaboração gráfica, layout, cores e dimensionamento dos textos e imagens foram desenvolvidos por profissional da área de design gráfico, quanto ao conteúdo textual, utilizou-se auxílio de profissional com formação em Letras/Português para revisão.

\section{RESULTADOS E DISCUSSÃO}

Após a revisão de literatura, os conteúdos foram elencados na seguinte ordem: Exame físico, Necessidades Humanas Básicas e Títulos Diagnósticos de Enfermagem, de acordo com o Guia para o Exame Físico do Sistema Respiratório materializado.

\section{Sessão 1 - Preparo Inicial}

$\mathrm{Na}$ sessão 1 denominada preparo inicial, procurou-se evidenciar as primeiras atividades do profissional que antecedem a entrevista e o exame físico de enfermagem. Para Jarvis ${ }^{(17)}$, trata-se de um momento essencial para realização do exame, pois é nesse momento que o profissional

\section{Quadro 1. Livros contemplados na revisão de literatura. Vitória, ES, Brasil, 2019}

\begin{tabular}{|l|c|c|}
\hline TíTULO & AUTORES & ANO \\
\hline Semiologia médica & Celmo Celene Porto & 2019 \\
\hline Fundamentos de Enfermagem & $\begin{array}{c}\text { Anne Griffin Perry e Patricia A. Potter, Amy M. Hall, } \\
\text { Patricia A. Stockert. }\end{array}$ & 2018 \\
\hline Fundamentos de enfermagem. Introdução ao processo de enfermagem & Mary Ellen Murray | Leslie D. Atkinson & 2017 \\
\hline Prática de Enfermagem & Sandra M. Nettina & 2017 \\
\hline Tratado de enfermagem Médico-Cirúrgico & Hinkle,Janice L., Cheever,Kerry H & 2017 \\
\hline Guia de Exame Físico para Enfermagem & Carolyn Jarvis & 2016 \\
\hline Procedimentos e competências de Enfermagem & Anne Griffin Perry e Patricia A. Potter. & 2015 \\
\hline Exame físico na prática clínica de enfermagem & Maria de Fatima Paula & 2015 \\
\hline Anamnese e exame físico: avaliação e diagnóstico de enfermagem adulta & Alba Lúcia Bottura Leite de Barros & 2015 \\
\hline Fundamentos de enfermagem Básica & Lois White, Gena Ducan e Wendy Baumle & 2012 \\
\hline Semiologia Médica: As bases do diagnóstico clínico & Mario Lopez, J. Laurentys Medeiros. & 2001 \\
\hline Processo de Enfermagem & Wanda de Aguiar Horta & 1979 \\
\hline
\end{tabular}


prepara o ambiente, separa os materiais, se apresenta e explica o procedimento ao paciente que será submetido ao exame. No entanto, estudos mostram que os enfermeiros têm negligenciado essa etapa, o que reduz a qualidade do exame ${ }^{(14,17)}$. Outros estudos evidenciam a importância desse momento, uma vez que o preparo inicial pré-exame físico corrobora para a execução da entrevista de enfermagem e o exame físico, onde ambos constituem a primeira etapa do processo de enfermagem, essencial para a operacionalização das atividades do enfermeiro ${ }^{(18,19)}$.

\section{Sessão 2 - Identificação do Paciente}

A sessão 2 do guia tratou da identificação do paciente, elencando itens de relevância para o serviço de saúde, tais como: nome completo, data de nascimento, endereço, motivo da internação dentro outros. Sabe-se que, de acordo com o Programa Nacional de Segurança do Paciente (PNSP), a identificação do paciente é fundamental para garantir a segurança e qualidade da assistência de enfermagem, uma vez que a literatura tem apontado falhas nesse processo ${ }^{(20)}$.

O instrumento desenvolvido encontra-se na Figura 1 abaixo, nele foram elencadas as sessões discutidas nesta pesquisa.

$\mathrm{O}$ acolhimento favorece a abertura de canais de comunicação, fortalecendo o vínculo e a comunicação terapêutica entre examinador e paciente. Quanto à identificação do paciente, sabe-se que esta resulta em uma importante conduta do enfermeiro, visto que previne possíveis eventos adversos que possam gerar danos ao paciente ${ }^{(21,22)}$.

\section{Sessão 3 - Entrevista de Enfermagem}

A sessão 3 do instrumento proposto aborda a entrevista de enfermagem preconizada pela Resolução COFEN n. ${ }^{\circ}$ $358 / 2009$, como ação privativa e fundamental para operacionalização do Processo de Enfermagem, composto pelas seguintes etapas: coleta de dados com-

\section{Figura 1. Guia para o Exame Físico do Sistema respiratório. Vitória, ES, Brasil, 2019}

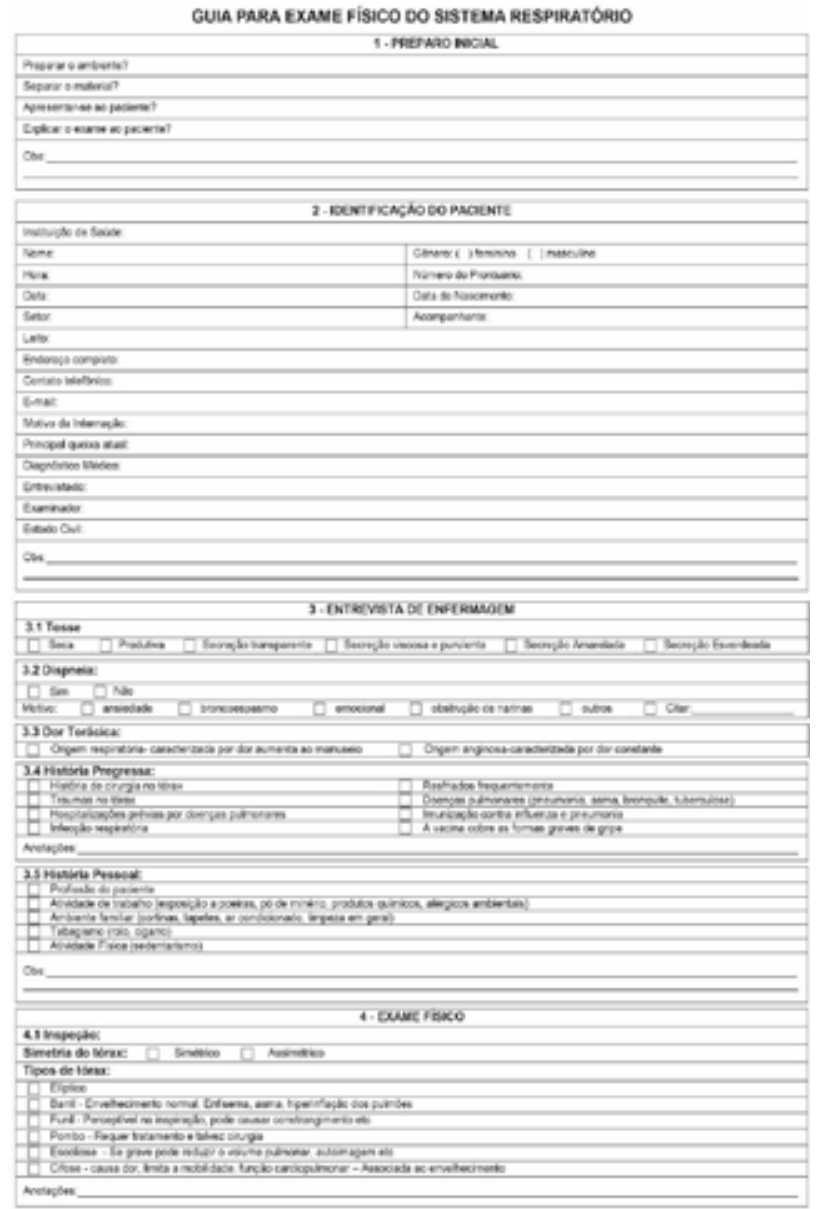

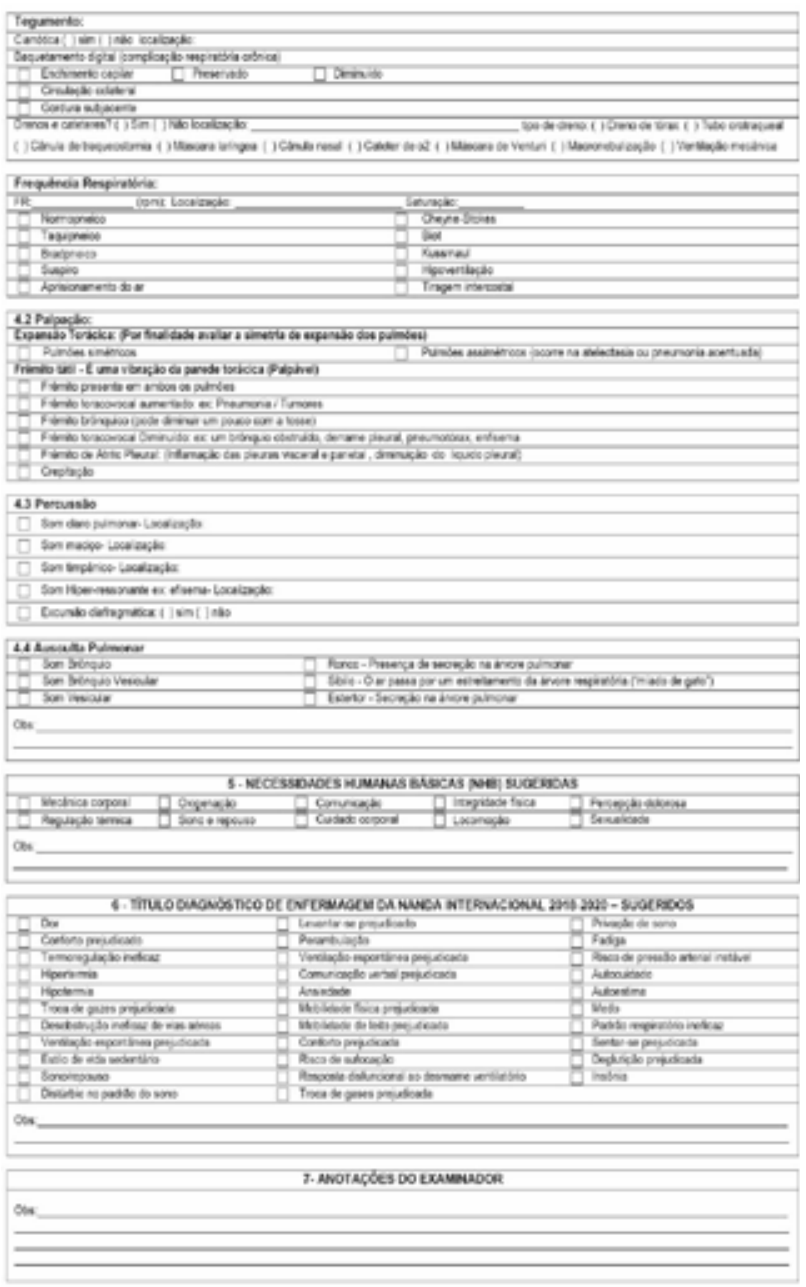


posta pela entrevista e o exame físico de enfermagem, diagnóstico, planejamento, implementação e avaliação ${ }^{(9)}$.

Entende-se que a entrevista de enfermagem permite ao enfermeiro identificar necessidades humanas básicas, parte fundamental para elencar os diagnósticos de enfermagem representativos, desta forma, a entrevista é subjetiva, ou seja, o que o paciente relata sobre si mesmo ${ }^{(3,9)}$.

Um estudo qualitativo e descritivo, que avaliou a narrativa de vinte gestantes e quatro enfermeiras a respeito da consulta de enfermagem em uma unidade básica de saúde, constatou o aumentou do grau de satisfação das usuárias, destacando-se o acolhimento como peça fundamental para coleta de dados ${ }^{(19)}$. No presente instrumento de avaliação do aparelho respiratório, objetivou-se identificar dados voltados para o histórico de tosse, dispneia, dor torácica, história pregressa e pessoal do paciente examinado ${ }^{(23)}$.

\section{Sessão 4 - Exame Físico}

A sessão 4 corresponde ao exame físico, que é subdivido pelas 4 técnicas propedêuticas semiológicas: inspeção, palpação, percussão e ausculta. São achados pautados em evidências, uma vez que as técnicas são realizadas para obter achados anormais, através dos sinais e sintomas já investigados anteriormente, descritos na entrevista de enfermagem. A realização do exame físico favorece o conhecimento e oportuniza acurácia na identificação das reais $\mathrm{NHB}$ do paciente, com isso, o enfermeiro vivencia nas suas atividades a sensação de maior autonomia para cuidar dos pacientes ${ }^{(2,3)}$.

Procura-se, na inspeção do tórax, encontrar achados anormais por meio da observação. Para tanto, é importante observar os seguintes aspectos: simetria, tipos de tórax, e entre os achados podemos ter o elíptico, o barril que está associado ao enfisema, asma, hiperinflação dos pulmões, tórax cifose, escoliose, pombo entre outros $^{(24)}$. Cabe também ao enfermeiro avaliar o tegumento do paciente, sabendo que a pele é o maior órgão do corpo humano e de grande importância de avaliação. Nela podemos encontrar sinais caracterís- ticos de possíveis doenças, como a cianose, que é um achado que pode representar a dificuldade de perfusão sanguínea, o baquetamento de digital que também é significante na inspeção pode representar um problema de respiração grave e precisa de investigação, os outros achados podem ser enchimento capilar, circulação colateral, gordura subjacente. Inspeciona-se também os dispositivos que o cliente faz uso, como drenos e cateteres ${ }^{(17,25)}$.

\section{Cabe também ao} enfermeiro avaliar

o tegumento do paciente, sabendo que a pele é o maior órgão do corpo humano e de grande importância de avaliação.

Ainda na técnica de inspeção, avalia-se a frequência respiratória com a visualização do tórax do cliente, sendo possível observar se ele está com uma frequência adequada ou não, e classificar como: normopneico, taquipneico, bradpneico, suspiro, aprisionamento de ar, cheyne-stock, biof, kussmaul, hipoventilação e tiragem intercostal. É importante também observar se ele faz uso de musculatura acessória, como por exemplo a tiragem intercostal ou de fúrcula supra- esternal e respiração freno-labial, achados assim levam o enfermeiro a identificar alterações, como exemplo a doença pulmonar obstrutiva crônica
(DPOC), que tem tratamento e acomete em sua maioria os fumantes, por si, utiliza de musculatura acessória devido à obstrução crônica gerada ${ }^{(25,26)}$.

$\mathrm{Na}$ palpação, se utiliza o tato, um dos cinco sentidos, para a avaliação. $\mathrm{O}$ sistema nervoso central (SNC) reconhece esse sentido e consegue diferenciar o quente e o frio e textura da pele. Existem técnicas para identificar achados na palpação. A expansão torácica tem por finalidade avaliar a simetria de expansão dos pulmões e podem ser encontrados achados como simétricos e assimétricos (atelectasia ou pneumonia acentuada). Já o frêmito tátil é uma vibração palpável do tórax, onde o enfermeiro sobrepõe as mãos ao tórax do paciente e pede para ele falar 33 e identifica através do tato se o frêmito está presente em ambos os pulmões, como o achado se o frêmito toracovocal está aumentado pode-se ter por diagnóstico a pneumonia ou tumores, já se o frêmito toracovocal tiver diminuído, pode estar relacionado a um brônquio obstruído, derrame pleural, pneumotórax, enfisema, frêmito brônquico, frêmito de atrito pleural e crepitações. A percussão é a terceira técnica utilizada no exame físico respiratório, são golpes feitos com os dedos e reproduzidos sons que podem ser: claro pulmonar, maciço, timpânico, hiper ressonante, excursão diafragmática ${ }^{(25,26)}$.

$\mathrm{Na}$ técnica de ausculta do tórax utiliza-se um aparelho denominado de estetoscópio para verificação de sons pulmonares, por exemplo, estes favorecem possíveis achados roncos, sibilos, estertores e atrito pleural, quando encontrados requerem do enfermeiro tomada de decisão ${ }^{(24)}$.

\section{Sessão 5 - Necessidades Humanas Básicas}

A sessão 5 aborda a temática da teoria das Necessidades Humanas Básicas (NHB) descritas por Wanda de Aguiar Horta, que foi uma grande influenciadora do ensino da assistência de enfermagem no Brasil. As NHB, descritas por ela, têm por finalidade avaliar de forma holística e individual o cliente, uma vez que essa teoria destaca que os indivíduos possuem necessidades universais que variam de um para 
o outro, com isso, a teoria possibilita listar as necessidades apresentadas e um maior vínculo entre enfermeiros e cliente ${ }^{(27)}$.

De acordo com Horta ${ }^{(28)}$, o paciente está em constante vulnerabilidade e, com isso, necessita de cuidados de enfermagem, as Necessidades Humanas Básicas (NHB) quando não atendidas podem gerar desconfortos que, quando prolongados, podem originar doenças passiveis da assistência de enfermagem. Desta forma, as NHB são agrupadas da seguinte forma: psicobiologias, psicossocial e psicoespiritual, que está relacionado ao sono, repouso, regulamentação térmica, mecânica corporal, sexualidade, percepção dolorosa, locomoção, cuidado corporal, oxigenação, comunicação, entre outros ${ }^{(29)}$.

A teoria da NHB possibilita de maneira concisa a criação de instrumento para o trabalho da assistência de enfermagem, pois as teorias reafirmam o cuidado prestado pela enfermagem, trazendo cientificidade e visibilidade a profissão. Esse modelo conceitual elaborado por Horta se fundamenta na Teoria da Motivação Humana de Maslow, que tem como base o conceito de hierarquia das necessidades que influenciam o comportamento humano ${ }^{(28,29)}$.

\section{Sessão 6 - Títulos Diagnósticos de En- fermagem na NANDA-I (2018-2020)}

$\mathrm{Na}$ sessão 6 do instrumento elaborado pelos autores, abordam-se os Títulos Diagnósticos da Nanda Internacional 2018-2020, sugeridos como parte inicial que irão compor os diagnósticos de enfermagem. O diagnóstico de enfermagem é uma atividade privativa do enfermeiro, o que reafirma a Lei do Exercício Profissional. O enfermeiro tem total autonomia e conhecimento para realizar o processo de enfermagem e utilizar de uma taxonomia própria de sua profissão, uma vez que na Resolução COFEN n. ${ }^{\circ}$ 358/2009 reafirma a aplicação da Sistematização da Assistência de Enfermagem e a execução Processo de enfermagem (PE). Para título diagnóstico deste instrumento de coleta de dados, utilizou-se a taxonomia da NANDA-I, que viabiliza o cuidado gerando maior autonomia e cientificidade ao enfermeiro, neste instrumento, utilizou-se os seguintes diagnósticos relacionados ao aparelho respiratório: dor, conforto, sono, ansiedade, fatiga dentre outros ${ }^{(9)}$.

\section{Sessão 7 - Anotações}

A sessão 7 destina-se ao campo de anotações do examinador, uma vez que pode haver achados importantes não contemplados no presente instrumento, cabe ressaltar que o mesmo ainda não foi submetido ao processo de validação por enfermeiros especialistas da área. A versão final do instrumento possui duas páginas, com perguntas abertas e fechadas.

\section{CONSIDERAÇÕES FINIAIS}

A elaboração de tecnologias na área da enfermagem é crescente, o enfermeiro se depara diariamente com pacientes apresentando alterações do aparelho respiratório. $\mathrm{O}$ produto gerado dessa pesquisa foi a elaboração de um instrumento que servirá como um guia para estudantes de enfermagem e enfermeiros.

Ressalta-se que a tecnologia construída se trata de uma versão beta, ou seja, necessita de novas abordagens no sentido de validação de conteúdo, clareza e relevância para a comunidade científica. Novos estudos são necessários para identificação de tecnologias voltadas ao aparelho respiratório, visto que a literatura apresenta poucos achados referente a temática.

\section{REFERÊNCIAS}

1. Arbex MA, et al. A poluição do ar e o sistema respiratório. J. bras. Pneumol [Internet].20120ct[acessoem29nov2019];38(5):643655. Disponivel em: http://www.scielo.br/scielo.php?script=sci arttext\&pid=S1806-37132012000500015\&lng=en\&nrm=iso.

2. Silva CMC, Teixeira ER. Exame físico e sua integralização ao processo de enfermagem na perspectiva da complexidade. Esc. Anna Nery [Internet]. 2011 Dec [acesso em 29 nov 2019];15(4):723729. Disponivel em: http://www.scielo.br/scielo.php?script=sci_ arttext\&pid=S1414-81452011000400010\&Ing=en\&nrm=iso.

3. Santos N, Veiga P, Andrade R. Importância da anamnese e do exame físico para o cuidado do enfermeiro. Rev. bras. enferm. [Internet]. 2011 Apr [acesso em 29 nov 2019];64(2):355-358. Disponivel em: http://www.scielo.br/scielo.php?script=sci_arttext\&pid=S0034-71672011000200021\&lng=en\&nrm=iso.

4. Corpolato RC, et al. Standardization of the duty shift in a General Adult Intensive Care Unit. Rev. Bras. Enferm. [Internet]. 2019 Feb [acesso em 29 nov 2019];72(Supl.1):88-95. Disponivel em: http://www.scielo.br/scielo.php?script=sci_arttext\&pi$\mathrm{d}=$ S0034-71672019000700088\&lng=en\&nrm=iso.
5. Pereira FGF, et al. Construção de um aplicativo digital para o ensino de sinais vitais. Rev. Gaúcha Enferm. [Internet]. 2016 [acesso em 29 nov 2019];37(2):e59015. Disponível em: http://www.scielo.br/scielo.php?script=sci_arttext\&pi$\mathrm{d}=$ S1983-14472016000200414\&lng=en\&nrm=iso.

6. Novaes HMD, Elias FTS. Uso da avaliação de tecnologias em saúde em processos de análise para incorporação de tecnologias no Sistema Único de Saúde no Ministério da Saúde. Cad. Saúde Pública [Internet]. 2013 [acesso em 29 nov 2019];29(Supl.1):S7-S16. Disponivel em: http://www.scielo.br/scielo.php?script=sci_arttext\&pid=S0102-311X2013001300002\&lng=en\&nrm=iso.

7. Lima SGG, Brito C, Andrade CJC. O processo de incorporação de tecnologias em saúde no Brasil em uma perspectiva internacional. Ciênc. saúde coletiva [Internet]. 2019 May [acesso em 29 nov 2019];24(5):1709-1722. Disponível em: http://www.scielo.br/scielo.php?script=sci_arttext\&pi$\mathrm{d}=\mathrm{S} 1413-81232019000501709 \& \operatorname{lng}=\mathrm{en} \& \mathrm{nrm}=$ iso.

8. Costa AC, Silva JV. Representações sociais da sistematização da assistência de enfermagem sob a ótica de en- 


\section{REFERÊNCIAS}

fermeiros. Rev. Enf. Ref. [Internet]. 2018 mar [acesso em 29 nov 2019];serIV(16):139-146. Disponivel em: http:/l www.scielo.mec.pt/scielo.php?script=sci_arttext\&pi$\mathrm{d}=$ S0874-02832018000100014\&lng=pt\&nrm=iso.

9. Conselho Federal de Enfermagem (BR). Resolução n. ${ }^{3}$ 358, 15 de outubro de 2009 [Internet]. Brasília (DF): COFEN, 2009 [acesso em 22 out 2019]. Disponivel em: http://www.cofen.gov.br/ resoluo-cofen-3582009_4384.html.

10. Garcia TR. Sistematização da assistência de enfermagem: aspecto substantivo da prática profissional. Esc. Anna Nery [Internet]. 2016 mar [acesso em 29 nov 2019];20(1):5-10. Disponivel em: http://www.scielo.br/scielo.php?script=sci_arttext\&pi$\mathrm{d}=\mathrm{S} 1414-81452016000100005 \& \operatorname{lng}=e n \& n r m=$ iso.

11. Benedet $S A$, et al. $O$ modelo de profissionalismo na implementação do Processo de Enfermagem (19792004). Rev. Bras. Enferm. [Internet]. 2018 ago [acesso em 02 dez 2019];71(4):1907-1914. Disponível em: http://www.scielo.br/scielo.php?script=sci_arttext\&pi$d=$ S0034-71672018000401907\&lng=en\&nrm=iso.

12. Boaventura AP, Santos PA, Duran ECM. Conocimiento teórico-práctico del enfermero del Proceso de Enfermería y Sistematización de Enfermería. Enferm. glob. [Internet]. 2017 [acesso em 03 dez 2019];16(46):182-216. Disponível em: http://scielo.isciii.es/scielo.php?script=sci_arttext\&pi$d=$ S1695-61412017000200182\&lng=es\&nrm=iso.

13. Araujo JL, et al. App móvel para processo de enfermagem em uma unidade de cuidado intensivo neonatal. Texto contexto - enferm. [Internet]. 2019 [acesso em 02 dez 2019];28:e20180210. Disponivel em: http://www.scielo.br/scielo.php?script=sci_arttext\&pid=S0104-07072019000100366\&lng=en\&nrm=iso.

14. Ramalho Neto JM, Fontes WD, Nobrega MML. Instrumento de coleta de dados de enfermagem em Unidade de Terapia Intensiva Geral. Rev. bras. enferm. [Internet]. 2013 Aug [acesso em 02 dez 2019];66(4):535-542. Disponível em: http://www.scielo.br/scielo.php?script=sci_arttext\&pid=S0034-71672013000400011\&lng=en\&nrm=iso.

15. Silva DVA, et al. Diagnósticos de enfermagem em programa domiciliar: mapeamento cruzado e Taxonomia da NANDA-I. Rev. Bras. Enferm. [Internet]. 2019 Jun [acesso em 02 dez 2019];72(3):584-591. Disponivel em: http://www.scielo.br/scielo.php?script=sci_arttext\&pi$d=$ S0034-71672019000300584\&lng=en\&nrm=iso.

16. Lima DVM. Desenhos de pesquisa: uma contribuição ao autor. Online braz. J. nurs. (Online) [Internet]. 2011 abr/ago [acesso em 02 dez 2019];10(2). Disponivel em: http://www.objnursing. uff.br/index.php/nursing/article/viewFile/3648/pdf.

17. Jarvis C. Guia de Exame Físico para Enfermagem. Rio de Janeiro: Elsevier; 2016.

18. Domingos CS, et al. Adaptation of software with the nursing process for innovation units. Rev. Bras. Enferm. [Internet]. 2019 Apr [acesso em 04 dez 2019];72(2):400-407. Disponível em: http://www.scielo.br/scielo.php?script=sci_arttext\&pi$\mathrm{d}=$ S0034-71672019000200400\&lng=en\&nrm=iso.

19. Gomes CBA, et al. Prenatal nursing consultation: narratives of pregnant women and nurses. Texto contexto - enferm. [Internet]. 2019 [acesso em 04 dez 2019];28:e20170544. Disponivel em: http://www.scielo.br/scielo.php?script=sci_arttext\&pid=S0104-07072019000100320\&lng=en\&nrm=iso.

20. Ministério da Saúde (BR). Portaria n. ${ }^{\circ} 529$, de $1^{\circ}$ de abril de 2013. Institui o Programa Nacional de Segurança do Paciente (PNSP) [Internet]. Brasília (DF): MS, 2013 [acesso em 29 nov 2019]. Disponível em: http://bvsms.saude.gov.br/bvs/saudelegis/gm/2013/prt0529_01_04_2013.html.

21. Martins ACT, et al. O Projeto AcolheSUS na Atenção Primária em Saúde do Distrito Federal, Brasil. Ciênc. saúde coletiva [Internet]. 2019 jun [acesso em 04 dez 2019];24(6):2095-2103. Disponivel em: http://www.scielo.br/scielo.php?script=sci_arttext\&pid=S1413-81232019000602095\&lng=en\&nrm=iso.

22. Behrens R. Segurança do paciente e direitos do usuário. Rev. Bioét. [Internet]. 2019 jun [acesso em 04 dez 2019];27(2):253260. Disponível em: http://www.scielo.br/scielo.php?script=sci_ arttext\&pid=S1983-80422019000200253\&Ing=en\&nrm=iso.

23. Porto CC. Semiologia médica. Ed. 8. Rio de Janeiro: Guanabara Koogan;2019.

24. Santos MAP, Dias LMD, Gonzaga MFN. O Papel do Enfermeiro no Exame Físico do Sistema Respiratório: Semiologia e Semiotécnica. Rev. Saúde em Foco [Internet]. 2018 [acesso em 04 dez 2019];10. Disponivel em: http://portal.unisepe.com.br/uni$\mathrm{fia} / \mathrm{wp}$-content/uploads/sites/10001/2018/06/047_O_PAPEL_DO_ENFERMEIRO_NO_EXAME_F\%C3\%8DSICO_DO_SISTEMA_RESPIRAT\%C3\%93̈RIO.pdf.

25. Barros ALBL. Anamnese e exame físico: avaliação diagnóstica de enfermagem no adulto. Porto Alegre: Artmed; 2016.

26. Marcos $L$, et al. Classificação da doença pulmonar obstrutiva crônica pela radiografia do tórax. Radiol Bras. 2013 Dec [acesso em 04 dez 2019];46(6):327-332. Disponível em: http://www.scielo.br/scielo.php?script=sci_arttext\&pi$\mathrm{d}=$ S0100-39842013000600327\&lng=en\&nrm=iso.

27. Lucena ICD, Barreira IA. Revista de enfermagem em novas dimensões: Wanda Horta e sua contribuição para a construção de um novo saber da enfermagem (1975-1979). Texto contexto enferm. [Internet]. 2011 Sep [acesso em 04 dez 2019];20(3):534540. Disponivel em: http://www.scielo.br/scielo.php?script=sci_ arttext\&pid=S0104-07072011000300015\&lng=en\&nrm=iso.

28. Horta WA. Processo de Enfermagem. Rio de Janeiro: Guanabara Koogan; 1979.

29. Neves RS. Sistematização da assistência de enfermagem em unidade de reabilitação segundo o modelo conceitual de horta. Rev. bras. enferm. [Internet]. 2006 Aug [acesso em 04 dez 2019];59(4):556-559. Disponivel em: http://www.scielo.br/scielo.php?script=sci_arttext\&pi$d=$ S0034-71672006000400016\&lng=en\&nrm=iso. 